\title{
Influence of molecular diffusion on alignment of vector fields - Eulerian analysis
}

\section{Gonzalez}

Received: date / Accepted: date

\begin{abstract}
The effect of diffusive processes on the structure of passive vector and scalar gradient fields is investigated by analyzing the corresponding terms in the orientation and norm equations. Numerical simulation is used to solve the transport equations for both vectors in a two-dimensional, parameterized model flow. The study highlights the role of molecular diffusion in the vector orientation process, and shows its subsequent action on the geometric features of vector fields.
\end{abstract}

Keywords Passive vector · Scalar gradient · Molecular diffusion · Alignment properties

\section{Introduction}

Transport of vectors in fluid flows is closely connected to the properties and to the very structure of velocity fields and scalar fields. It is therefore a basic issue in fluid dynamics. In addition, it is relevant to many fields, including microfluidics, process engineering, combustion, turbulent mixing, mixing protocols, geophysical flows, astrophysical flows, etc. However, the kinematics of vectors undergoing the mechanical action of a velocity field is not that simple; it may even be very intricate when an active vector such as vorticity, the magnetic field, or the gradient of a non-passive scalar is considered [1-3]. In three-dimensional flows, even the characteristics of the gradient of a passive scalar bear the stamp of the interaction between strain and vorticity [4].

As far as inertial processes - inviscid vortex stretching, advection of tracers, kinematic dynamo - are considered, the purely mechanical aspect can be investigated in itself by dropping viscous and diffusive effects while keeping the rich phenomenology unchanged. This approach, however, does not include the full mechanical process; as viscosity and molecular diffusion are felt beyond their respec-

M. Gonzalez

CNRS, UMR 6614/CORIA, Site universitaire du Madrillet

76801 Saint-Etienne du Rouvray, France

E-mail: michel.gonzalez@coria.fr 
tive length scales, there is a range of scales where the mechanics of transported vectors is itself actually not free from the viscous and/or the diffusive influence.

More specifically, viscous and diffusive effects not only limit the growth of vectors norm, but also affect their geometric features such as orientation in the strain basis, which in turn determines the production of norm as well as the structure of the vector field. In this respect, viscosity was shown to directly influence vorticity alignment (see [5] and references therein) and vortex lines geometry [6,7]. In two-dimensional turbulence, the role of molecular diffusion on the orientation dynamics of scalar gradient and vorticity gradient was analyzed by Lapeyre et al. [8]; Protas et al. [9] studied the Reynolds-number dependence of the vorticity gradient alignment. In three-dimensional turbulence, the effects of both the Reynolds and Schmidt numbers on the alignment of the scalar gradient were examined by Vedula et al.[10]. Brandenburg et al. [11] addressed the influence of the magnetic diffusivity on the alignment of flux lines of the magnetic field.

Viscous and diffusive effects, however, still deserve special attention. On the theoretical level, especially, a better understanding of small-scale phenomena is needed. One also has to face the problem of molecular processes when modelling vector and tensor fields [12-14]. In this study, the influence of molecular diffusion on the geometric characteristics and structure of passive vector and scalar gradient fields is scrutinized by directly considering the relevant terms in the orientation and norm equations. Plainly, the latter have remained unexplored, for it seems that a few studies tackled the question in this way [6-8]. In passing, only Constantin et al. [7] analyzed the diffusive terms by splitting them into a linear, Laplacian part, and a non-linear part resulting from norm/orientation interaction; this was for vorticity in special, basic cases.

The present work, then, specifically addresses the following questions: regarding the alignment mechanism, how strong are diffusive processes as compared with the mechanical action of the flow? What is the overall action of molecular diffusion in this respect? Is there any connection between diffusive processes and flow structure? How great are diffusive non-linear terms in comparison to linear ones? Do the passive vector and the scalar gradient differ from one another in all these respects?

The equations for the orientation and norm of the passive vector and of the scalar gradient are given in Section 2. The two-dimensional model flow and the numerical method are described in Sections 3 and 4, respectively. Section 5 is devoted to the discussion of the numerical results, and conclusions are drawn in Section 6 .

\section{Vector field equations}

\subsection{Equations for the passive vector}

The general, Eulerian equation for a passive vector, B, in an incompressible flowfield, $u$, is:

$$
\frac{\partial \mathrm{B}}{\partial t}+\mathrm{u} \cdot \nabla \mathrm{B}=\mathrm{A} \cdot \mathrm{B}+D \Delta \mathrm{B}
$$

where $\mathrm{A}$ is the velocity gradient tensor, and $D$ is a diffusion coefficient. The magnetic field evolution in a flow is given by Eq. (1) as well. The passive vector is 
actually a good model of the magnetic field in the kinematic regime [15] - i.e. when the magnetic field is too weak to backreact on the flowfield through the Lorentz force.

Orientation and norm are more suitable than Cartesian coordinates for analyzing a vector field. This formalism uses invariants - the vector orientation in the strain eigenframe and the vector norm -, and is connected to the structure of the vector field. This approach was used to study the fine structure of the scalar gradient field and small-scale mixing mechanisms $[16,17]$. Vector B is defined by its norm, $B$, and its orientation, $\theta$, in the fixed frame of reference as: $\mathrm{B}=B(\cos \theta, \sin \theta)$. The Eulerian equations for $B$ and $\theta$ are derived from Eq. (1):

$$
\begin{gathered}
\frac{\partial B}{\partial t}+\text { u. } \nabla B=\frac{\sigma}{2} \sin [2(\theta+\Phi)] B+D \Delta B-D|\nabla \theta|^{2} B, \\
\frac{\partial \theta}{\partial t}+\text { u. } \nabla \theta=\frac{1}{2}\{\omega+\sigma \cos [2(\theta+\Phi)]\}+D \Delta \theta+2 D \frac{\nabla B . \nabla \theta}{B},
\end{gathered}
$$

where $\sigma=\left(\sigma_{n}^{2}+\sigma_{s}^{2}\right)^{1 / 2}$ is the strain intensity, with $\sigma_{n}=\partial u / \partial x-\partial v / \partial y$ and $\sigma_{s}=\partial u / \partial y+\partial v / \partial x$, the normal and shear strain components, respectively; $\omega=$ $\partial v / \partial x-\partial u / \partial y$ is the vorticity and $\Phi$, given by $\tan (2 \Phi)=\sigma_{n} / \sigma_{s}$, is the orientation of the strain principal axes in the fixed frame of reference, $(x, y)$.

Special alignments of the passive vector are $\theta_{d}=-\Phi+\pi / 4$ (extensional strain direction), for which the norm growth rate reaches its maximum value, $\sigma / 2$, and $\theta_{c}=-\Phi-\pi / 4$ (compressional strain direction), for which it takes the minimum value $-\sigma / 2$.

In Eqs. (2) and (3), convection is represented by the second term on left-hand side. In addition to convection, these equations show the effects of the flow and of molecular diffusion on the vector norm and orientation. The action of the flow consists of the straining of the vector norm - first term, denoted by $\mathcal{A}(B)$, on the right-hand side of Eq. (2) -, and changes in angle $\theta$ due to strain and rotation first term, denoted by $\mathcal{A}(\theta)$, on the right-hand side of Eq. (3).

Diffusive terms split up into linear, Laplacian terms, and non-linear terms. The former, $D \Delta B=\mathcal{D}_{l}(B)$ and $D \Delta \theta=\mathcal{D}_{l}(\theta)$, express diffusive smoothing; the latter, $-D|\nabla \theta|^{2} B=\mathcal{D}_{n l}(B)$ and $2 D(\nabla B . \nabla \theta) / B=\mathcal{D}_{n l}(\theta)$, express dissipation caused by angle gradients, and diffusive tilting resulting from the interaction between norm gradient and orientation gradient. Note that the complete diffusive terms, $\mathcal{D}_{l}(B)+\mathcal{D}_{n l}(B)$ and $\mathcal{D}_{l}(\theta)+\mathcal{D}_{n l}(\theta)$, are the components of the vectorial Laplacian $D \Delta \mathrm{B}$ along the directions parallel and orthogonal to vector $\mathrm{B}$, respectively [6]. Diffusive terms clearly make the analytic approach for the orientation and norm equations $[16,18]$ untractable in a realistic flow. Moreover, with molecular diffusion, the universal nature of local equilibrium alignments, strictly determined by the local velocity gradient tensor, is lost.

While there is a simple one-way coupling between the norm and the orientation of a non-diffusive vector - the straining of the vector depends on its alignment in the strain eigenframe -, molecular diffusion brings about a tighter interaction. Term $\mathcal{D}_{n l}(B)$ shows that molecular diffusion strongly wipes out the vector field in disorganized regions, where large angle gradients prevail. In addition, diffusive tilting - expressed by $\mathcal{D}_{n l}(\theta)$ - should be weak for the largest vectors. As a result, molecular diffusion is likely to arrange the vector field so that disorganized regions correspond to small vector norm, while alignment with the largest vectors is promoted by diffusive smoothing of angle in their vicinity [7]. 
2.2 Equations for the gradient of a scalar

In an incompressible flow, the equation for the gradient, $G=\nabla \Theta$, of a scalar $\Theta$ is:

$$
\frac{\partial \mathrm{G}}{\partial t}+\mathrm{u} \cdot \nabla \mathrm{G}=-\mathrm{A}^{T} \cdot \mathrm{G}+D \Delta \mathrm{G}
$$

and the equations in terms of norm and orientation of vector $\mathrm{G}=G(\cos \theta, \sin \theta)$ are:

$$
\begin{gathered}
\frac{\partial G}{\partial t}+\text { u. } \nabla G=-\frac{\sigma}{2} \sin [2(\theta+\Phi)] G+D \Delta G-D|\nabla \theta|^{2} G, \\
\frac{\partial \theta}{\partial t}+\text { u. } \nabla \theta=\frac{1}{2}\{\omega-\sigma \cos [2(\theta+\Phi)]\}+D \Delta \theta+2 D \frac{\nabla G \cdot \nabla \theta}{G} .
\end{gathered}
$$

The diffusive terms take the same form as for the passive vector. The action of the flow on the scalar gradient, however, is different; this time, the maximum norm growth rate $\sigma / 2$ is reached for $\mathrm{G}$ parallel with the compressional strain direction, and the minimum growth rate $-\sigma / 2$ for $G$ parallel with the extensional strain direction.

\section{Model flow}

The flow belongs to the family of flows defined by Tanner and Hughes [19]. It is a combination of the Galloway-Proctor, circularly polarized, unsteady flow [20], with a steady, two-dimensional, cat's-eye-type flow [21]. It is specialized to a twocomponent velocity field, namely $(u, v, w)=(\partial \psi / \partial y,-\partial \psi / \partial x, 0)$, with the general form of the streamfunction, $\psi$, given by:

$$
\psi=\alpha\{\cos [y+\cos (t)]+\sin [x+\sin (t)]\}+\delta[\cos (y)-\sin (x)],
$$

where $\alpha=\sqrt{3 / 2-\delta^{2}}$. When $\delta$ spans the range $[0, \sqrt{3 / 2}]$ the flow characteristics are continuously shifted from those of the circularly polarized flow to those of the cat's-eye flow. The structure of these flowfields was thoroughly analyzed by Galloway [22] and Courvoisier et al. [23], respectively - see also references therein. This family of parameterized flows was also used to study the role of alignment dynamics in the growth of a diffusionless passive vector [24]. Strain intensity, vorticity, and orientation of strain principal axes are easily obtained from the velocity field resulting from the streamfunction defined by Eq. (7).

The study was made for $\delta=0$ - unsteady, Galloway-Proctor flow. Figure 1 displays the velocity field and the Okubo-Weiss parameter, $Q=\sigma^{2}-\omega^{2}$; the latter shows the flow structure in terms of hyperbolic $(Q>0)$ and elliptic $(Q<0)$ regions, for $\delta=\sqrt{3 / 2}$. When $\delta=0$ the flowfield has a similar, but unsteady spatial structure.

\section{Numerical solution}

Equations (1) and (4) were solved for components $B_{i}$ and $G_{i}$, respectively. The latter were subsequently used to derive the orientation and norm of vectors B and G. The angle derivatives were not computed directly, but from the vector components derivatives. The numerical domain was a $2 \pi \times 2 \pi$ square with periodic boundary 


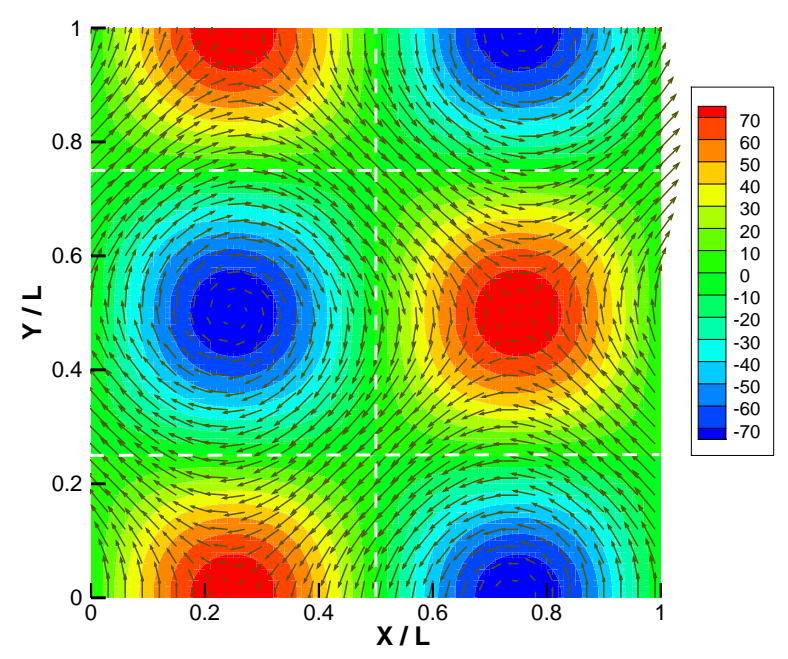

Fig. 1 Okubo-Weiss parameter, $Q$, and velocity field for $\delta=\sqrt{3 / 2}$; the white dashed lines on which $Q=0$ - separate the hyperbolic from the elliptic regions of the flow.

conditions. As the computation of diffusive terms needed good accuracy, the finite difference scheme was sixth-order in space [25]. The resolution was explicit in time.

The initial conditions for B and $\mathrm{G}$ were:

$$
B_{1}(x, y, 0)=\sin y \quad ; \quad B_{2}(x, y, 0)=\cos x
$$

and

$$
G_{1}(x, y, 0)=\sin x \quad ; \quad G_{2}(x, y, 0)=\cos y .
$$

The main parameter for analyzing the structure and evolution of vector $\mathrm{G}$ is the Péclet number, $P e=U L / D$, where $U$ and $L$ are a velocity- and a lengthscale, respectively. For vector $\mathrm{B}$, this number is denoted by $R e_{B}$ which is reminiscent of the magnetic Reynolds number when $B$ is a model for the magnetic induction. In this study, $L=2 \pi$ and $U=\sqrt{3}$ - the value around which the maximum velocity within the field oscillates. The convective timescale, $L / U$, is denoted by $T_{c}$. All quantities, then, are nondimensional.

To keep up the vector fields, a forcing term, $f_{\mathrm{B}} \mathrm{B}$ (resp. $f_{\mathrm{G}} \mathrm{G}$ ), was added to Eq. (1) [resp. Eq. (4)]. This term affects the norm equation, but not the orientation equation. The stabilizing value of $f_{\mathrm{B}}$ (resp. $f_{\mathrm{G}}$ ) is a priori unknown and was thus derived by trial and error.

\section{Results and discussion}

Four values of $R e_{B}$ (resp. $P e$ ), namely 10, 20, 40, and 80, were considered in the unsteady flow. The mesh size was $150 \times 150,200 \times 200,300 \times 300$, and $400 \times 400$, respectively. 
Figures 2 to 5 show the statistics for the passive vector. The smallest vectors $\left(B^{2}<10^{-3}\right)$ are discarded, but this hardly affects the statistical results. Figure 2 shows the statistics of $\mathcal{R}_{1}=\left|\mathcal{D}_{n l}(B)\right| /\left(|\mathcal{A}(B)|+\left|\mathcal{D}_{n l}(B)\right|\right)$; this ratio compares the destruction rate of norm, due to the non-linear diffusive term, to the straining rate. Unconditioned spatial averaging as well as averaging conditioned on small $-\left\langle\mathcal{R}_{1} \mid B<\langle B\rangle\right\rangle$ - and large $\left.-\left\langle\mathcal{R}_{1} \mid B\right\rangle\langle B\rangle\right\rangle$ - vector norm is achieved over the computational domain at each time step. The magnitude of $\left|\mathcal{D}_{n l}(B)\right|$ relative to straining, expressed by ratio $\mathcal{R}_{1}$, clearly grows on average as $R e_{B}$ is decreased. In addition, it is greater for small vectors, which is consistent with the finding of Constantin et al. [7] that in non-aligned regions - large $|\nabla \theta|$ - vector norm should be small.
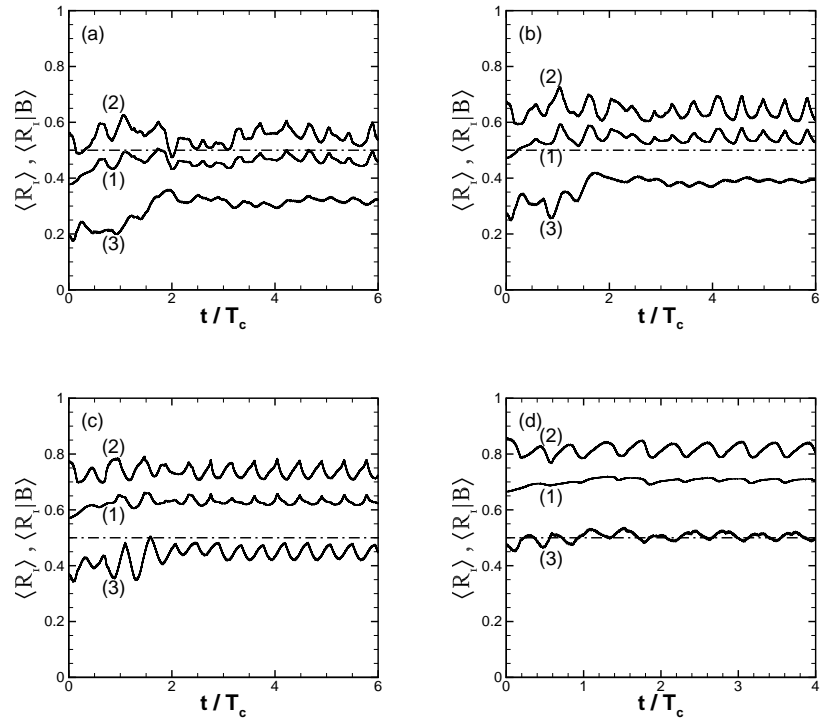

Fig. 2 Spatial average of $\mathcal{R}_{1}$ (passive vector); (a) $R e_{B}=80$; (b) $R e_{B}=40$; (c) $R e_{B}=20$; (d) $R e_{B}=10$; (1) unconditioned averaging; (2) $\left\langle\mathcal{R}_{1} \mid B\langle\langle B\rangle\rangle ;(3)\left\langle\mathcal{R}_{1} \mid B\right\rangle\langle B\rangle\right\rangle$; the dash dotted line indicates statistically comparable values of $\left|\mathcal{D}_{n l}(B)\right|$ and $|\mathcal{A}(B)|$.

The diffusive terms of the orientation equation [Eq. (3)] are investigated through ratios $\mathcal{R}_{2}=\left|\mathcal{D}_{t}(\theta)\right| /\left(|\mathcal{A}(\theta)|+\left|\mathcal{D}_{t}(\theta)\right|\right)$ - with $\mathcal{D}_{t}(\theta)=\mathcal{D}_{l}(\theta)+\mathcal{D}_{n l}(\theta)-, \mathcal{R}_{3}=$ $\left|\mathcal{D}_{n l}(\theta)\right| /\left(|\mathcal{A}(\theta)|+\left|\mathcal{D}_{n l}(\theta)\right|\right)$, and $\mathcal{R}_{4}=\left|\mathcal{D}_{n l}(\theta)\right| /\left(\left|\mathcal{D}_{l}(\theta)\right|+\left|\mathcal{D}_{n l}(\theta)\right|\right)$. Figure 3 shows that both $\mathcal{R}_{2}$ and $\mathcal{R}_{3}$ grow as $\operatorname{Re}_{B}$ is decreased, which was expectable. For $R e_{B}=80, \mathcal{R}_{2}$ fluctuates within the range $0.3-0.4$. Even for this value of $R e_{B}$, then, the diffusive rates are far from negligible in comparison to the mechanical rate resulting from strain and rotation. Interestingly, $\mathcal{R}_{4}$ fluctuates around 0.4 , which shows that in the orientation equation the linear and the non-linear diffusive rates are of the same order of magnitude; the former just slightly exceeds the latter on average. Since $\left\langle\mathcal{R}_{2}\right\rangle<\left\langle\mathcal{R}_{3}\right\rangle$, terms $\mathcal{D}_{l}(\theta)$ and $\mathcal{D}_{n l}(\theta)$ also tend to oppose each other. 

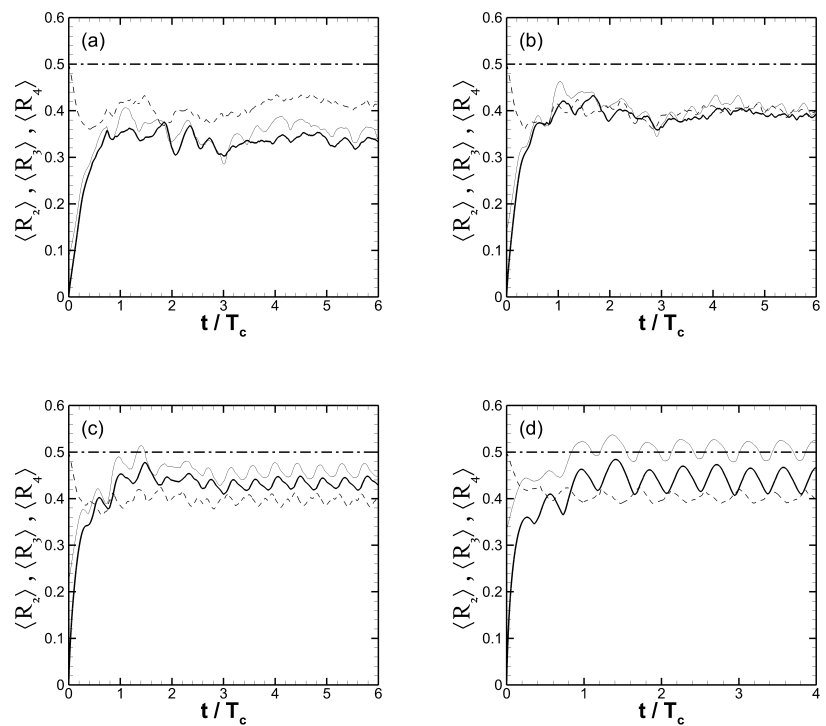

Fig. 3 Spatial averages of $\mathcal{R}_{2}$ - bold solid line,$- \mathcal{R}_{3}$ - thin solid line -, and $\mathcal{R}_{4}$ - dashed line - (passive vector); (a) $R e_{B}=80$; (b) $R e_{B}=40$; (c) $R e_{B}=20$; (d) $R e_{B}=10$.

In Fig. 4, the diffusive tilting rate corresponding to the non-linear term $\mathcal{D}_{n l}(\theta)$ is compared to the mechanical rate $\mathcal{A}(\theta)$ while conditioning on either small or large vectors. Clearly, diffusive tilting resulting from the interaction between norm gradient and orientation gradient is more efficient for small vectors than for large ones. This result, too, agrees with the analysis of Constantin et al. [7]. The total diffusive tilting, $\mathcal{D}_{l}(\theta)+\mathcal{D}_{n l}(\theta)$, displays the same trends (and is thus not shown), consistently with the study of Lapeyre et al. [8] in two-dimensional turbulence. Figure 4 also shows that the ratio of non-linear diffusive rate to mechanical rate is significant for small vectors whatever the value of $\operatorname{Re}_{B}$; indeed $\left\langle\mathcal{R}_{3} \mid B<\langle B\rangle\right\rangle \sim 0.5$ at least. Large vectors, by contrast, need strong diffusion to feel diffusive tilting; $\left.\left\langle\mathcal{R}_{3} \mid B\right\rangle\langle B\rangle\right\rangle$ grows from $\sim 0.2$ to $0.4-0.5$ as $R_{B}$ is decreased from 80 to 10 .

Finally, statistics conditioned on local flow structure show that the total diffusive tilting rate - when measured against the mechanical rate - is greater in strain regions than in rotation regions. This is displayed in Fig. 5 which shows $\left\langle\mathcal{R}_{2} \mid Q>0\right\rangle$ and $\left\langle\mathcal{R}_{2} \mid Q<0\right\rangle$ for $\operatorname{Re}_{B}=20,40$, and 80 .

Similar results were derived for the scalar gradient, as shown in Figs. 6 to 8 which must be compared with Figs. 2, 4, and 5, respectively.

As regards the effect of molecular diffusion on vector orientation, the statistics derived in this simple flow for a passive vector and the gradient of a scalar bear out the major conclusions of the few studies which addressed this question [7, 8]. Disorganized regions - large angle gradient - correspond to small vectors, and diffusive tilting is weaker for large vectors than for small ones. This gives support to the idea that molecular diffusion promotes the alignment of the largest vectors. 

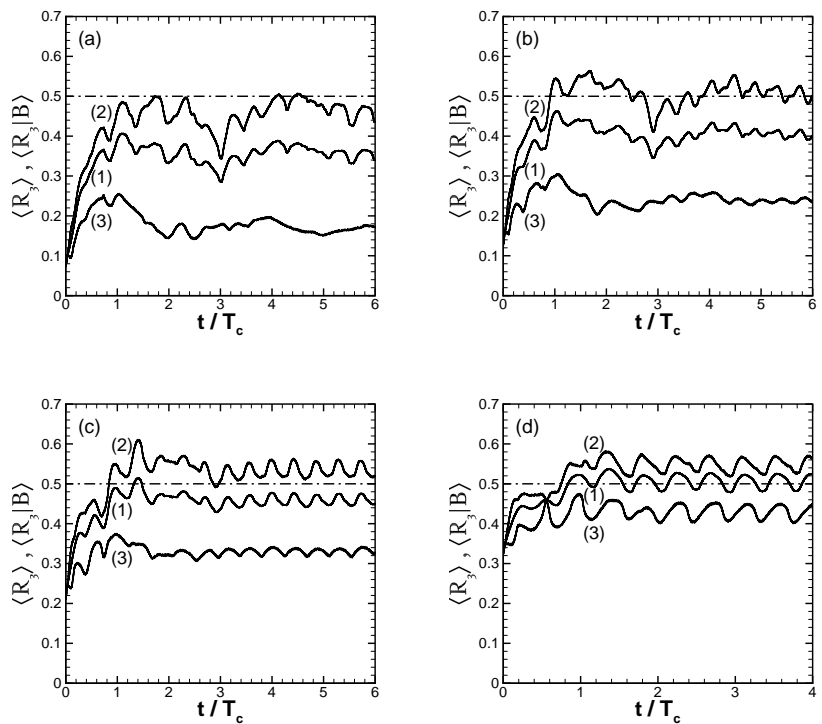

Fig. 4 Spatial average of $\mathcal{R}_{3}$ (passive vector); (a) $R e_{B}=80$; (b) $R e_{B}=40$; (c) $R e_{B}=20$; (d) $\operatorname{Re}_{B}=10$; (1) unconditioned averaging; (2) $\left\langle\mathcal{R}_{3} \mid B<\langle B\rangle\right\rangle$; (3) $\left.\left\langle\mathcal{R}_{3} \mid B\right\rangle\langle B\rangle\right\rangle$; the dash dotted line indicates statistically comparable values of $\left|\mathcal{D}_{n l}(\theta)\right|$ and $|\mathcal{A}(\theta)|$.
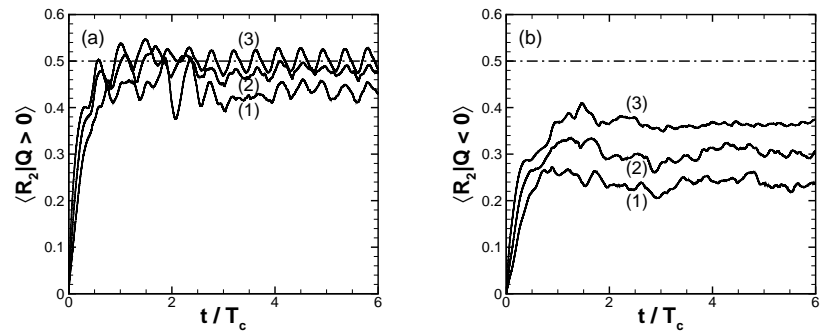

Fig. 5 Spatial average of $\mathcal{R}_{2}$ conditioned on the local structure (passive vector); (a) prevailing strain; (b) prevailing rotation; (1) $R e_{B}=80 ;(2) R e_{B}=40 ;(3) R e_{B}=20$; the dash dotted line indicates statistically comparable values of $\left|\mathcal{D}_{t}(\theta)\right|$ and $|\mathcal{A}(\theta)|$.

\section{Conclusion}

The influence of molecular diffusion on vector alignment was analyzed for a passive vector and for the gradient of a scalar. For both of them, molecular diffusion is expressed by two types of terms in the norm and orientation equations, namely linear Laplacian terms, and non-linear terms resulting from the interaction between norm gradient and angle gradient. 

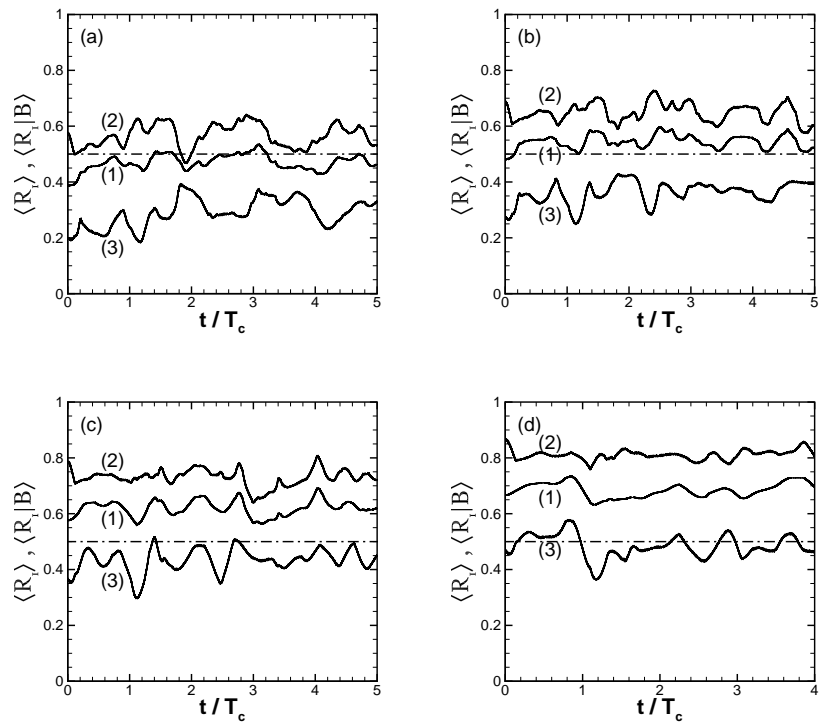

Fig. 6 Spatial average of $\mathcal{R}_{1}$ (scalar gradient); (a) $P e=80$; (b) $P e=40$; (c) $P e=20$; (d) $P e=10$; (1) unconditioned averaging; (2) $\left\langle\mathcal{R}_{1}\right| G\langle\langle G\rangle\rangle$; (3) $\left.\left\langle\mathcal{R}_{1} \mid G\right\rangle\langle G\rangle\right\rangle$; the dash dotted line indicates statistically comparable values of $\left|\mathcal{D}_{n l}(G)\right|$ and $|\mathcal{A}(G)|$.
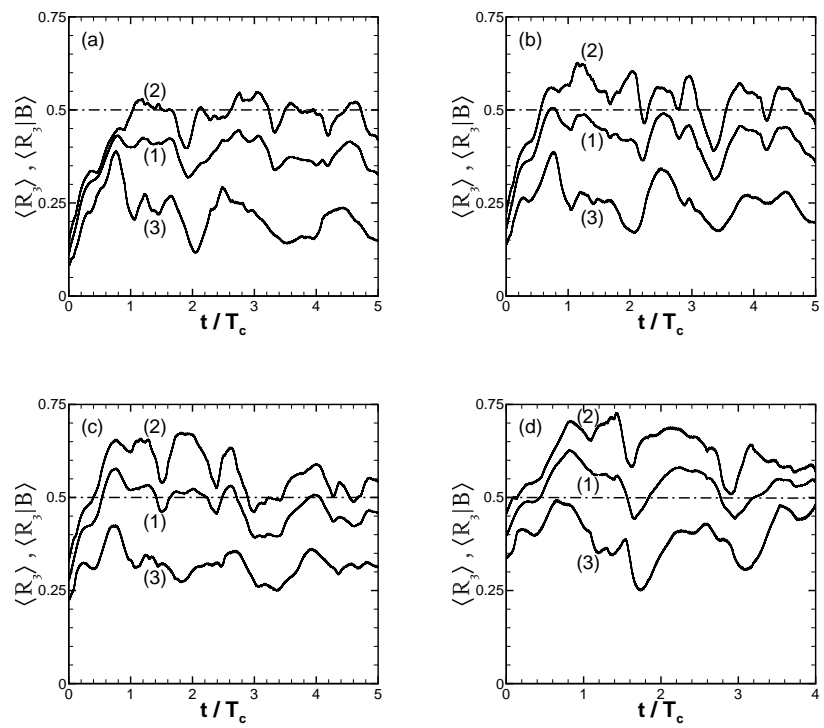

Fig. 7 Spatial average of $\mathcal{R}_{3}$ (scalar gradient); (a) $P e=80$; (b) $P e=40$; (c) $P e=20$; (d) $P e=10$; (1) unconditioned averaging; (2) $\left\langle\mathcal{R}_{3}\right| G\langle\langle G\rangle\rangle$; (3) $\left.\left\langle\mathcal{R}_{3} \mid G\right\rangle\langle G\rangle\right\rangle$; the dash dotted line indicates statistically comparable values of $\left|\mathcal{D}_{n l}(\theta)\right|$ and $|\mathcal{A}(\theta)|$. 

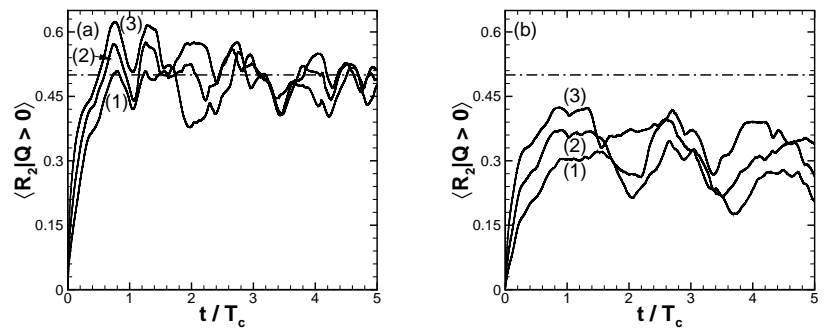

Fig. 8 Spatial average of $\mathcal{R}_{2}$ conditioned on the local structure (scalar gradient); (a) prevailing strain; (b) prevailing rotation; (1) $P e=80$; (2) $P e=40$; (3) $P e=20$; the dash dotted line indicates statistically comparable values of $\left|\mathcal{D}_{t}(\theta)\right|$ and $|\mathcal{A}(\theta)|$.

These diffusive processes were numerically investigated in a parameterized flowfield. In this study, the flow was an unsteady, Galloway-Proctor flow. Although the model flowfield was analytic and specialized to a two-dimensional case, it included the essential features of local flow structure, and was shown to be useful for exploring the physics of transported vectors.

Specifically regarding the influence of molecular diffusion, the following results were derived for the passive vector and the scalar gradient alike:

- overall, the total diffusive tilting is far from negligible as compared with the mechanical action of the flow on vector orientation. Even for the highest value of $R_{B}$ (and $P e$ ) tested, the diffusive tilting rate amounts to $30 \%$ of the total rate of angle variation;

- disorganized regions - large angle gradients - are correlated with small vectors. This feature directly results from the strong lessening of vector norm caused by molecular diffusion where angle gradients are large, and is significant whatever the value of $R_{B}$ or $P e$ considered in the study;

- large vectors are less affected by diffusion-induced angle variations than small ones. This result, together with the previous one, shows that molecular diffusion promotes the alignment of the largest vectors, in agreement with the findings of previous studies.

A further analysis of the diffusive terms of the orientation equation also showed that:

- when measured against the flow mechanical action, the effect of molecular diffusion on vector orientation is greater in strain regions than in rotation regions;

- on average, the linear and the non-linear terms are of the same order of magnitude; the former just slightly exceeds the latter;

- these terms do not reinforce, but rather tend to oppose each other.

The findings of this study are valid for $R e_{B}$ and $P e$ numbers ranging from small to moderate values, which is relevant to several types of flow. As part of the results are consistent with previous numerical simulations of two-dimensional turbulence, one can surmise that the conclusions of this work might also hold in turbulence. 
Finally, the study suggests new challenges for including this physics in the modelling of transported vectors. In a future work, a further insight could be brought by analyzing the influence of molecular diffusion on the alignment dynamics, especially if considered from a Lagrangian view.

\section{References}

1. Lüthi, B., Tsinober, A., Kinzelbach, W.: Lagrangian measurements of vorticity dynamics in turbulent flow. J. Fluid Mech. 528, 87-118 (2005)

2. Favier, B., Bushby, P.J.: Small-scale dynamo action in rotating compressible convection. J. Fluid Mech. 690 262-287 (2012)

3. Diamessis, P.J., Nomura, K.K.: Interaction of vorticity, rate-of-strain, and scalar gradient in stratified homogeneous sheared turbulence. Phys. Fluids 12, 1166-1188 (2000)

4. Brethouwer, G., Hunt, J.C.R., Nieuwstadt, F.T.M.: Micro-structure and Lagrangian statistics of the scalar field with a mean gradient in isotropic turbulence. J. Fluid Mech. 474, 193-225 (2003)

5. Holzner, M., Guala, M., Lüthi, B., Liberzon, A., Nikitin, N., Kinzelbach, W., Tsinober, A.: Viscous tilting and production of vorticity in homogeneous turbulence. Phys. Fluids 22061701 (2010)

6. Kida, S., Takaoka, M.: Breakdown of frozen motion of vorticity field and vorticity reconnection. J. Phys. Soc. Jpn. 60, 2184-2196 (1991)

7. Constantin, P., Procaccia, I., Segel, D.: Creation and dynamics of vortex tubes in threedimensional turbulence. Phys. Rev. E 51, 3207-3222 (1995)

8. Lapeyre, G., Hua, B.L., Klein, P.: Dynamics of the orientation of active and passive scalars in two-dimensional turbulence. Phys. Fluids 13, 251-264 (2001)

9. Protas, B., Babiano, A., Kevlahan, N.K.-R.: On geometrical alignment properties of twodimensional forced turbulence. Physica D 128, 169-179 (1999)

10. Vedula, P., Yeung, P.K., Fox, R.O.: Dynamics of scalar dissipation in isotropic turbulence: a numerical and modelling study. J. Fluid Mech. 433, 29-60 (2001)

11. Brandenburg, A., Procaccia, I., Segel, D.: The size and dynamics of magnetic flux structures in magnetohydrodynamic turbulence. Phys. Plasmas 2, 1148-1156 (1995)

12. Jeong, E., Girimaji, S.S.: Velocity-gradient dynamics in turbulence: Effect of viscosity and forcing. Theor. Comput. Fluid Dyn. 16, 421-432 (2003)

13. Meneveau, C.: Lagrangian dynamics and models of the velocity gradient tensor in turbulent flows. Annu. Rev. Fluid Mech. 43, 219-245 (2011)

14. Gonzalez, M.: Kinematic properties of passive scalar gradient predicted by a stochastic Lagrangian model. Phys. Fluids 21, 055104 (2009)

15. Moffat, K.: The amplification of a weak applied magnetic field by turbulence in fluids of moderate conductivity. J. Fluid Mech. 11, 625-635 (1961)

16. Lapeyre, G., Klein, P., Hua, B.L.: Does the tracer gradient align with strain eigenvectors in 2D turbulence? Phys. Fluids 11, 3729-3737 (1999)

17. Garcia, A., Gonzalez, M., Paranthoën, P.: On the alignment dynamics of a passive scalar gradient in a two-dimensional flow. Phys. Fluids 17, 117102 (2005)

18. Garcia, A., Gonzalez, M.: Analysis of passive scalar gradient alignment in a simplified three-dimensional case. Phys. Fluids 18, 058101 (2006)

19. Tanner, S.E.M., Hughes, D.W.: Fast-dynamo action for a family of parameterized flows. Astrophys. J. 586, 685-691 (2003)

20. Galloway, D.J., Proctor, M.R.E.: Numerical calculations of fast dynamos in smooth velocity fields with realistic diffusion. Nature 356, 691-693 (1992)

21. Childress, S., Soward, A.M.: Scalar transport and alpha-effect for a family of cat's-eye flows. J. Fluid Mech. 205, 99-133 (1989)

22. Galloway, D.J.: ABC flows then and now. Geophys. Astrophys. Fluid Dyn. 106, 450-467 (2012)

23. Courvoisier, A., Gilbert, A.D., Ponty, Y.: Dynamo action in flows with cat's-eyes. Geophys. Astrophys. Fluid Dyn. 99, 413-429 (2005)

24. Gonzalez, M.: Effect of orientation dynamics on the growth of a passive vector in a family of model flows. Fluid Dyn. Res. 46, 015510 (2014)

25. Lele, S.K.: Compact finite difference schemes with spectral-like resolution. J. Comput. Phys. 103, 16-42 (1992) 